\title{
Methods for Research Evidence Synthesis: The Scoping Review Approach
}

\author{
Heidi Sucharew, $\mathrm{PhD}^{1,2 *}$; Maurizio Macaluso, MD, $\mathrm{DrPH}^{1,2}$
}

'Division of Biostatistics and Epidemiology, Cincinnati Children's Hospital Medical Center, Cincinnati, Ohio; ${ }^{2}$ Department of Pediatrics, University of Cincinnati College of Medicine, Cincinnati, Ohio.

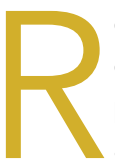

esearch evidence synthesis involves the aggregation of available information using well-defined and transparent methods to search, summarize, and interpret a body of literature, frequently following a systematic review approach. A scoping review is a relatively new approach to evidence synthesis and differs from systematic reviews in its purpose and aims. ${ }^{1}$ The purpose of a scoping review is to provide an overview of the available research evidence without producing a summary answer to a discrete research question. ${ }^{2}$ Scoping reviews can be useful for answering broad questions, such as "What information has been presented on this topic in the literature?" and for gathering and assessing information prior to conducting a systematic review. ${ }^{1}$

In this issue of the Journal of Hospital Medicine, Fan et al. used a scoping review to identify information available in the literature on contributors to loss and theft of controlled drugs in hospitals and the safeguards that have been suggested to address these diversions. ${ }^{3}$ The authors followed Arksey and O'Malley's framework for scoping reviews and the PRISMA-ScR (Preferred Reporting Items for Systematic reviews and Meta-Analyses extension for Scoping Reviews) checklist in reporting findings. ${ }^{2,4}$

\section{PURPOSE OF A SCOPING REVIEW}

Scoping reviews describe existing literature and other sources of information and commonly include findings from a range of different study designs and methods. ${ }^{5}$ The broad scope of the collected information makes using formal meta-analytic methods difficult, if not impossible. Results of a scoping review often focus on the range of content identified, and quantitative assessment is often limited to a tally of the number of sources reporting a particular issue or recommendation. In contrast, systematic reviews commonly select the information sources by requiring specific study types, such as randomized controlled trials, and imposing quality standards, such as adequate allocation concealment, and place their emphasis on synthesizing data to address a specific research question. (Table) By focusing on specific studies, the synthesis component in a systematic review often takes the form of a meta-analysis in which the results of multiple scientific studies are combined to develop a summary conclusion, such as a common effect

*Corresponding Author: Heidi Sucharew, PhD; E-mail: heidi.sucharew@cchmc. org; Telephone: 513-803-1920.

Published online first June 12, 2019.

Received: May 2, 2019; Revised: May 14, 2019; Accepted: May 15, 2019

() 2019 Society of Hospital Medicine DOI 10.12788/jhm.3248 estimate, along with an evaluation of its heterogeneity across studies.

A scoping review can be a particularly useful approach when the information on a topic has not been comprehensively reviewed or is complex and diverse. 6 Munn et al. proposed several objectives that can be achieved utilizing the scoping review framework, including identifying types of existing evidence in a given field, clarifying key concepts or definitions in the literature, surveying how research is conducted on a certain topic, identifying key characteristics related to a certain topic, and identifying knowledge gaps. ${ }^{1}$ When choosing to use a scoping review approach, it is important that the objective of the review align with the review's indication or purpose.

\section{METHODOLOGICAL FRAMEWORK OF SCOPING REVIEWS}

Scoping reviews, like systematic reviews, require comprehensive and structured searches of the literature to maximize the capture of relevant information, provide reproducible results, and decrease potential bias from flawed implementations. The methodological framework for scoping reviews was developed by Arksey and O'Malley' and further refined by Levac et al. ${ }^{7}$ and the Joanna Briggs Institute. ${ }^{6,8}$ Arksey and O'Malley's framework for scoping reviews consists of the following six steps:

- Step 1: Identify the research question-the research question should be clearly defined and usually broad in scope to provide extensive coverage.

- Step 2: Identify relevant studies-the search strategy should be thorough and broad in scope and typically include electronic databases, reference lists, hand searches, and gray literature (ie, substantive or scholarly information that has not been formally published and often is not peer-reviewed), including conference abstracts, presentations, regulatory data, working papers, and patents.

- Step 3: Study selection-the study selection process can include post hoc, or modified, inclusion and exclusion criteria as new ideas emerge during the process of gathering and reviewing information.

- Step 4: Chart the data-the data extraction process in a scoping review is called data charting and involves the use of a data charting form to extract the relevant information from the reviewed literature.

- Step 5: Collate, summarize, and report the results-the description of the scope of the literature is commonly presented in tables and charts according to key themes.

- Optional Step 6: Consultation exercise-in this optional 
TABLE. Characteristics of Systematic and Scoping Reviews

\begin{tabular}{lll}
\hline & Systematic Review & Scoping Review \\
\hline Purpose & Provide empirical evidence that meets prespecified criteria & Provide a narrative or descriptive account of available information \\
\hline Research question & Specific, focused on a single issue & Broadly defined \\
\hline Study protocol & A priori & A priori and post hoc \\
\hline Search strategy & Explicit and transparent & Explicit and transparent \\
\hline Study selection & Restricted to certain study types, meeting quality standards & All study types, nonstandard sources of information \\
\hline Data extraction & Developed at the protocol stage before the review is conducted & Informed by the review process, applied at the study selection stage \\
\hline Bias assessment & Well-defined process for extracting information relevant to evidence synthesis & Data charting according to key general themes \\
\hline Results & Mandatory critical appraisal & Optional (but desirable) \\
\hline
\end{tabular}

step, stakeholders outside the study review team are invited to provide their insights to inform and validate findings from the scoping review.

Since the number of studies included in a scoping review can be substantial, several study team members may participate in the review process. When multiple reviewers are employed, the team ought to conduct a calibration exercise at each step of the review process to ensure adequate interrater agreement. In addition, the PRISMA-ScR guidelines should be followed when reporting findings from scoping reviews to facilitate complete, transparent, and consistent reporting in the literature. ${ }^{4}$

\section{LIMITATIONS OF THE SCOPING REVIEW APPROACH}

The scoping review approach has several limitations. Scoping reviews do not formally evaluate the quality of evidence and often gather information from a wide range of study designs and methods. By design, the number of studies included in the review process can be sizable. Thus, a large study team is typically needed to screen the large number of studies and other sources for potential inclusion in the scoping review. Because scoping reviews provide a descriptive account of available information, this often leads to broad, less defined searches that require multiple structured strategies focused on alternative sets of themes. Hand searching the literature is therefore necessary to ensure the validity of this process. Scoping reviews do not provide a synthesized result or answer to a specific question, but rather provide an overview of the available literature. Even though statements regarding the quality of evidence and formal synthesis are avoided, the scoping review approach is not necessarily easier or faster than the systematic review approach. Scoping reviews require a substantial amount of time to complete due to the wide coverage of the search implicit in the approach.

Like other studies, scoping reviews are at risk for bias from different sources. Critical appraisal of the risk of bias in scoping reviews is not considered mandatory, but some scoping reviews may include a bias assessment. Even if bias is not for- mally assessed, that does not mean that bias does not exist. For example, selection bias may occur if the scoping review does not identify all available data on a topic and the resulting descriptive account of available information is flawed.

\section{WHY DID THE AUTHORS USE THE SCOPING REVIEW METHOD?}

Fan et al. used the scoping review approach to examine the available information on contributors to and safeguards against controlled-drug losses and theft (drug diversion) in the hospital setting. ${ }^{3}$ The authors addressed the following questions: (1) "What clinical units, health professions, or stages of the medication-use process are commonly discussed?" (2) "What are the identified contributors to diversion in hospitals?" and (3) "What safeguards to prevent or detect diversion in hospitals have been described?" Part of the rationale for using a scoping review approach was to permit the inclusion of a wide range of sources falling outside the typical peer-reviewed article. The authors comment that the stigmatized topic of drug diversion frequently falls outside the peer-reviewed literature and emphasize the importance of including such sources as conferences, news articles, and legal reports. The search strategy included electronic research databases, such as Web of Science, as well as an extensive gray literature search. Multiple reviewers were included in the process and a calibration exercise was conducted to ensure consistency in the selection of articles and to improve interrater agreement. The scoping review identified contributors to controlled-drug diversion and suggested safeguards to address them in the hospital setting.

\section{OTHER CONSIDERATIONS}

Methodological approaches to evidence synthesis vary, and new methods continue to emerge to meet different research objectives, including evidence mapping, ${ }^{9}$ concept analysis, ${ }^{10}$ rapid reviews, ${ }^{11}$ and others. ${ }^{12}$ Choosing the right approach may not be straightforward. Researchers may need to seek guidance from methodologists, including epidemiologists, statisticians, and information specialists, when choosing an appro- 
priate review approach to ensure that the review methods are suitable for the objectives of the review.

Disclosures: The authors have no conflicts of interest to disclose.

Financial Disclosures: The authors have no financial relationships relevant to this article to disclose.

\section{References}

1. Munn Z, Peters M, Stern C, Tufanaru C, McArthur A, Aromataris E. Systematic review or scoping review? Guidance for authors when choosing between a systematic or scoping review approach. BMC Med Res Methodol. 2018;18:143. doi: 10.1186/s12874-018-0611-x

2. Arksey $\mathrm{H}, \mathrm{O}$ 'Malley L. Scoping Studies: towards a methodological framework. Int J Soc Res Methodol. 2005;8(1):19-32. doi: 10.1080/1364557032000119616

3. Fan M, Tscheng D, Hamilton M, Hyland B, Reding R, Trbovich P. Diversion of controlled drugs in hospitals: a scoping review of contributors and safeguards. J Hosp Med. 2019;14(7):419-428. doi: 10.12788/jhm.3228

4. Tricco AC, Lillie E, Zarin W, et al. PRISMA Extension for Scoping Reviews (PRISMA-ScR): Checklist and Explanation. Ann Intern Med. 2018;169(7):
467-473. doi: 10.7326/M18-0850

5. Davis K, Drey N, Gould D. What are scoping studies? A review of the nursing literature. Int J Nurs Stud. 2009;46(10):1386-1400. doi: 10.1016/j. ijnurstu.2009.02.010

6. Peters MD, Godfrey CM, Khalil H, Mclnerney P, Parker D, Soares CB. Guidance for conducting systematic scoping reviews. Int J Evid Based Healthc. 2015;13(3):141-146. doi: 10.1097/XEB.00000000000000050.

7. Levac D, Colquhoun H, O'Brien KK. Scoping studies: advancing the methodology. Implement Sci. 2010;5(1):69. doi: 10.1186/1748-5908-5-69.

8. Peters MDJ, Godfrey C, Mclnerney P, Baldini Soares C, Khalil H, Parker D. Scoping reviews. In: Aromataris E, Munn Z, eds. Joanna Briggs Institute Reviewer's Manual. Adelaide, Australia: Joanna Briggs Inst; 2017. Available from https://reviewersmanual.joannabriggs.org/

9. Hetrick SE, Parker AG, Callahan P, Purcell R. Evidence mapping: illustrating an emerging methodology to improve evidence-based practice in youth mental health. J Eval Clin Pract. 2010;16(6):1025-1030. doi: 10.1111/j.13652753.2008.01112.x.

10. Ream E, Richardson A. Fatigue: a concept analysis. Int J Nurs Stud. 1996;33(5):519-529. doi: 10.1016/0020-7489(96)00004-1.

11. Tricco AC, Antony J, Zarin W, et al. A scoping review of rapid review methods. BMC Med. 2015;13(1):224. doi: 10.1186/s12916-015-0465-6.

12. Grant MJ, Booth A. A typology of reviews: an analysis of 14 review types and associated methodologies. Health Info Libr J. 2009;26(2):91-108. doi: 10.1111/j.1471-1842.2009.00848.x. 